\title{
Traduire
}

Revue française de la traduction

$236 \mid 2017$

Traducteurs en blouse blanche

\section{Adapter 50 Shades! The Musical pour la scène québécoise}

Alice Defacq

\section{CpenEdition}

Journals

Édition électronique

URL : http://journals.openedition.org/traduire/923

DOI : 10.4000/traduire.923

ISSN : 2272-9992

\section{Éditeur}

Société française des traducteurs

\section{Édition imprimée}

Date de publication : 1 juin 2017

Pagination : 75-84

ISSN : 0395-773X

\section{Référence électronique}

Alice Defacq, « Adapter 50 Shades! The Musical pour la scène québécoise », Traduire [En ligne],

236 | 2017, mis en ligne le 01 juin 2019, consulté le 30 juin 2019. URL : http://journals.openedition.org/ traduire/923 ; DOI : 10.4000/traduire.923 


\section{Adapter 50 Shades! The Musical pour la scène québécoise}

\section{Alice Defacq}

Il est aujourd'hui notoire d'affirmer que l'humour constitue pour le traducteur un problème sérieux, délicat même, dans la mesure où il l'invite à spéculer sur l'appréciation ou non des éléments comiques dans la culture cible. Dans la notion d'humour prédomine "le partage d'un rire ou d'un sourire, qui met principalement en jeu le langage et l'énonciation, tandis que le comique, qu'il soit de situation ou gestuel, peut passer (ou non) les frontières culturelles sans que n'intervienne la question de la traduction " (Tran-Gervat 2011 : 6). Les études sur l'humour foisonnent et montrent qu'il peut devenir un véritable casse-tête pour le traducteur se retrouvant " en grand danger de "s'arracher les cheveux" " (Soulier 1998: 12). Ce dernier doit identifier les éléments cocasses et décider de leur sort. "Plus généralement, le maître-mot en matière de traduction, c'est la décision" (Ladmiral 2004 : 19). Dès lors, face à ce problème, le traducteur peut être amené à réécrire le texte original afin de retrouver une formulation humoristique et de faire rire, à son tour, le public.

Voici les bases sur lesquelles nous nous proposons de greffer notre problématique laquelle envisagera l'adaptation du musica/(1) comme un exercice perfectible ayant pour objectif d'apporter un effet comique original au livret initial. Pour ce faire, nous nous appuierons sur 50 Shades! The musical [50 Shades ! La Parodie Musicale(2)] écrit en 2014 par Albert Samuels. C'est l'adaptation parodique du roman érotique 50 Shades of Grey (2011) d'E.L. James. Après avoir vu le spectacle à Los Angeles, Didier Morissonneau(3) décide d'en acheter les droits pour ensuite le produire au Québec. S'ensuit alors un travail de collaboration avec Amélie Veille(4), chargée d'adapter les chansons.

(1) Nous reprenons le terme anglais musical qui est l'abréviation de musical comedy, musical play et musical theater. Les Francophones emploient " comédie musicale " or il est difficile d'accoler cette étiquette à Sweeney Todd, Fiddler on the Roof ou Mamma Mia! qui sont respectivement un thriller musical, un musical drama et un jukebox musical.

(2) Les livrets n'ont pas fait l'objet de publication. Ce sont les adaptateurs eux-mêmes qui nous les ont confiés.

(3) Producteur et concepteur de spectacles connu au Québec pour ses musicals (Joe Dassin), ses productions théâtrales (Le Journal d'Anne Frank) et pour avoir présenté au Canada des artistes de variétés (Michel Sardou, Johnny Hallyday).

(4) Auteur-compositrice-interprète. Elle compte quatre albums à son actif et de nombreux succès radiophoniques. 
Précisons d'emblée que nous n'abordons pas ici le phénomène d'adaptation dans le sens d'une modification, c'est-à-dire la transposition d'un roman érotique en musical parodique, mais dans celui du passage de l'anglais au français. L'adaptation est donc un produit fini destiné à une création scénique. Morissonneau et Veille se placent dans deux optiques différentes : l'une énonciatrice et l'autre chantable. Ils cherchent à reproduire l'efficacité humoristique du texte source dans une langue cible pour un public d'une autre culture, tout en présentant un musical qui respecte la mise en scène de départ et ne trahisse pas la pensée musicale du compositeur. Dans cet article, nous nous trouvons alors au carrefour de plusieurs approches théoriques puisque le musical est un genre qui allie harmonieusement théâtre et numéros musicaux(5). Pour ce qui est de la partie théâtrale, nous nous adossons aux théories de Regattin afin de suggérer, sans prétention d'exhaustivité, certains éléments théoriques à propos de l'adaptation du musical pour la scène étrangère. En ce qui concerne la partie musicale, nous nous appuyons sur la stratégie Pentathlon de Low pour démontrer comment les contraintes de la forme peuvent guider et limiter les choix de l'adaptateur. Trois problèmes hétéroclites sont analysés : l'intertextualité, le décrochage des mots et le vocabulaire érotique.

Commençons par l'intertextualité qui renvoie, dans son acception la plus large, aux interactions intertextes pouvant prendre la forme de références, d'allusions ou de parodies. Afin de recréer une équivalence, le traducteur doit " posséder les connaissances littéraires et culturelles lui permettant de reconnaître la présence d'un texte dans un autre, mais il doit aussi faire preuve de la compétence critique nécessaire à la formulation de la signification de la relation intertextuelle " (Venuti 2006 : 17). Autrement dit, il navigue en eaux troubles car, outre le fait de devoir repérer les éléments intertextes drôles qui seront appréciés peu ou prou dans la culture cible, il doit également déterminer ce qui pourra être conservé. Traduire revient donc à produire un équivalent textuel de l'original tout en tenant compte des contraintes liées à la forme. N'oublions pas que nous travaillons sur un texte destiné à la scène, ce qui implique une compréhension immédiate de l'humour "où aucun retour en arrière n'est possible " (Seide 1982 : 60). Cela renvoie aux théories fondées sur le texte dramatique attestant de "l'immédiateté du discours théâtral [et] l'importance de la restitution des valeurs culturelles [...] pour en faciliter la compréhension " (Regattin 2004 : 160). Adapter pour la scène musicale demande au traducteur de prendre le risque de s'écarter du livret original afin d'obtenir un texte second efficace en termes de jouabilité(6) et de chantabilité. En d'autres termes, il opte pour une domestication du musical qui est une réduction ethnocentrique du texte étranger aux valeurs culturelles dominantes. Ladmiral préfère parler de traduction " cibliste " au sens où elle cible la culture d'arrivée, concept devenu canonique. II existe quasiment autant de terminologies

(5) "The musical number [can be] divided into dance numbers, duets, songs, etc. " (Di Giovanni 2008 : 299).

(6) Voir GREGORY William, 2012, "Jouabilité : un concept indéfinissable, incontournable... traduisible ou intraduisible?", in Traduire, 222, p. 7-21. 
que de théoriciens, mais toutes renvoient au désir de produire " une traduction lisible " (Venuti 1995 : 20). Si cette stratégie est controversée, elle se révèle un moyen efficient pour le musical:

1 CAROL. Well I have brought something that I think might pique the interest of the group. It's Martha Stewart's "Cooking Soup for One." (50S-LA p. 3-4(7))

1a JOHANNE. Eh bien, les filles... J'ai apporté un livre, qui, je crois, m'a l'air pas mal intéressant... C'est un nouveau livre de Ricardo : "Cuisiner de la soupe pour une personne " ! (50S-Q p. 5)

En l'occurrence, c'est la référence culinaire qui pose ici problème. Comme ce livre de cuisine est employé pour susciter le sourire, Morissonneau n'a d'autre ressource que de se livrer à un travail de réécriture. II fait donc appel à son imagination et trouve un équivalent intelligible par son public. L'adaptateur transpose l'efficacité humoristique de la tirade par l'insertion d'un référent culinaire qu'il suppose - à juste titre - connu des spectateurs. L'humour est par conséquent compris puisque le destinataire partage avec le destinateur un "imaginaire ethnosocioculturel collectif " (Boyer 2001: 37) reposant essentiellement sur le décryptage et la reconnaissance immédiate de l'élément culturel. Grâce à ce clin d'œil complice, la connivence entre celui qui parle et celui qui écoute est rétablie. Ce remplacement ne peut être perçu comme une trahison, mais comme une forme de fidélité par rapport au texte source puisque Morissonneau insuffle le désir des auteurs de déclencher le rire. Si le référent avait été préservé tel quel, il aurait engendré un malaise chez un public non réceptif et été, de ce fait, beaucoup moins drôle.

Ce changement n'a pas posé de réels problèmes à l'adaptateur car le référent en question apparaissait dans le dialogue. La tâche de Veille s'est néanmoins quelque peu compliquée lorsqu'elle s'est attelée au lyric (8). Depuis plusieurs années maintenant, des études sont venues remplir le champ de la recherche sur la traduction des chansons(9). Low marque un tournant dans ce domaine avec son Pentathlon Principle, " a deliberate balancing of five different criteria - singability, sense, naturalness, rhythm and rhyme. This balancing should be central to the overall strategy and also a guide to microlevel decision-making " (2005: 185). S'appuyant sur ce principe, Franzon distingue cinq choix de traduction :

(7) 50S-LA renvoie au livret de Los Angeles (2014) et 50S-Q à celui du Québec (2014). Nous respectons les normes typographiques des livrets : les dialogues sont en minuscule et les chansons en majuscule.

(8) Ce vocable est employé par Albert Willemetz pour désigner une technique d'écriture : l'adaptation de paroles sur une musique préexistante.

(9) Nous ne faisons pas référence aux études menées sur les chansons d'opéra ou de films musicaux, mais à celles effectuées sur les chansons en général. 
1. Leaving the song untranslated; 2. Translating the lyrics but not taking the music into account; 3. Writing new lyrics to the original music with no overt relation to the original lyrics; 4. Translating the lyrics and adapting the music accordingly - sometimes to the extent that a brand new composition is deemed necessary; 5. Adapting the translation to the original music (2008: 376).

Ces options semblent être théoriquement disponibles au traducteur alors qu'en réalité ce sont les exigences du metteur en scène qui l'amèneront à opérer tel ou tel type de stratégie. Dans 50 Shades! La Parodie musicale, Veille devra opter pour l'option cinq qui est, avouons-le, la plus difficile. Elle doit produire une traduction chantable qui prenne en compte la métrique musicale, c'est-à-dire les temps forts et les temps faibles, mais aussi la courbe mélodique. "Un intervalle mélodique ascendant a tendance à accentuer naturellement la syllabe qui se trouve en haut de l'intervalle, même si celle-ci se trouve sur un temps faible. Tout cela est absolument central et doit primer sur le sens littéral "(10). De plus, il faut qu'elle considère les éventuelles rimes et le nombre de syllabes qui sont "les deux éléments qui rendent ce travail si difficile "(11). La chanson "I don't make love" met bien en lumière les quelques difficultés rencontrées par Veille :

2 CHRISTIAN

YOU WANNA BE CUDDLIN' SNUGLLIN' WATCHIN' NETFLIX

BUT INSTEAD I LIKE TO BURN YOU WITH A VICE AROUND MY DICK! [...]

(Extended improvised section with audience)

You all came out to the matine

I'm gonna be inside your Puss-ey (50S-LA p. 26-27)

2a CHRISTIAN

TU VOUDRAIS TE BLOTTIR EN CUILLÈRE DEVANT LA TÉLÉ

MAIS JE PRÉFÈRE TE MENOTTER SUR LE LIT ET TE FOUETTER ! [ ... ]

(Ad lib de Christian)

Et si tu viens de Saint Félicien

J'vas passer la nuit dans ton vagin (50S-Q p. 30-31)

Christian chante à Anna qu'il préfère coucher avec elle plutôt que regarder la télévision. Les auteurs obtiennent un effet comique en associant Netflix avec dick. Ce jeu ne va pas se prêter facilement à la traduction.

"Cette chanson a été l'une des plus difficiles à faire. Quand j'ai écrit ce texte en 2014, je me suis dit qu'il y avait encore beaucoup de monde qui ne savait pas ce qu'était Netflix. J'aurais pu le garder mais ça ne bonifiait pas la phrase. En plus, c'était difficile de trouver des rimes en [Iks].

(10) Courriel du 23/02/2016 entre l'adaptateur Alain Perroux et l'auteur de l'article.

(11) Courriel du 02/12/2016 entre l'adaptateur Éric Taraud et l'auteur de l'article. 
Je pouvais le faire mais je voulais vraiment garder l'esprit de la chanson. Dans le fond, je raconte la même affaire car, dans les deux cas, Christian dit pourquoi il est devenu comme ça „(12).

Comme la référence n'évoque rien pour la majorité du public québécois et qu'elle pose des problèmes rimiques, Veille choisit l'option de la substitution sémantique ou hyperonymisante puisqu'elle remplace Netflix par télé. Ce gommage est radical mais efficace : I'auditoire n'est pas déconcentré, le contenu de la chanson reste inchangé et le travail de l'adaptateur en est facilité. En effet, elle dispose maintenant d'une large palette de mots se terminant en [e]. Elle propose alors fouetter, qui n'est indéniablement pas la traduction de dick, mais qui fut choisie pour les besoins du public. "Si j'avais gardé le mot à mot, j'aurais choqué les spectateurs québécois. Ce passage est violent et beaucoup trop vulgaire, donc je l'ai atténué "(13). Le rendu de ces lyrics découle de deux modifications : d'une part, le choix de la substitution pour répondre à une exigence rimique. "The making of singable translations is a very complex skopos because the target text must fit the pre-existing music "(Low 2005 : 185) ; d'autre part, l'atténuation de la vulgarité afin de ne pas heurter le public. Si Veille se livre à un léger travail de réécriture, ce n'est pas par pudeur, mais en raison de la perte rimique et de la grossièreté que le mot à mot aurait pu provoquer.

Outre la référence culturelle, ce qui nous intéresse en 2a c'est j'vas qui est la manière québécoise de dire je vais. Morissonneau et Veille ont inséré, dans le livret cible, un français régional pour ajouter un effet comique original. Expliquons-nous. 50 Shades! The Musical renferme une mise en abyme : trois lectrices se réunissent pour discuter de 50 Shades of Grey contant les aventures d'Ana et de Christian. Nous avons donc l'histoire des trois femmes qui s'enchâsse dans celle des personnages du livre. Dans le livret source, les acteurs des deux histoires dialoguent en anglais standard. Or, dans le livret cible, c'est une tout autre affaire : les lectrices parlent québécois tandis que les personnages du roman échangent en français. Nous sommes visiblement en présence de ce que nous pourrions qualifier de stratégies de traduction de la part des adaptateurs. Ces choix ne sont pas aléatoires mais prennent en compte le public cible. Examinons la première stratégie :

3 PAM. Oh my god, this one made me pregnant just looking at me.

BEV. Yeah! Tie me up, big daddy. (50S-LA p. 45)

3a VANESSA. Oh My God, heille lui j'pense qu'y m'a mis enceinte juste à me regarder dans les yeux.

SARAH. Heille le grand. Viens donc icitte m'attacher un peu. (50S-Q p. 50)

(12) Skype interview entre Amélie Veille et l'auteur de l'article le 29/12/2016.

(13) Idem. 
4 KATE. He put his ballsack inside of you?!

ANA. Oh, not his testicles silly! That would be gross. No. He put his silver ben wa balls inside of me, and spanked me. (50S-LA p. 48)

4a KATE. Il a mis ses testicules dans toi ?

ANA. Non, pas ses testicules! Franchement! Ses boules chinoises. II m'a mis des boules chinoises avant de me donner la fessée. (50S-Q p. 54)

Nous découvrons une différence nette entre les deux histoires sur scène. Le québécois, bien marqué en 3a, est totalement inexistant en 4a. Des mots comme heille, icitte, des anglicismes tels que oh my god et le remplacement de il par y signalent l'accent régional des lectrices. Quant au dialogue entre Ana et Christian, il a été simplement rendu en français. Le livret cible louvoie alors entre deux régiolectes, mais pourquoi?

"Au Québec, toutes les séries télévisées sont doublées en français. II y a quelques rares exceptions comme Les Pierrafeu et Les Simpsons qui ont été doublées en québécois. On grandit donc en voyant des émissions où des gens parlent "à la française". C'est une expression québécoise qui veut dire "parler comme un Français, un Parisien". Les personnages principaux de la pièce ne peuvent donc pas parler "à la française" si l'action se passe au Québec "(14).

L'insertion de deux français régionaux est d'abord guidée par un désir de cohérence avec le déplacement géographique adopté. L'idée des auteurs est d'utiliser la scène comme un miroir pour renvoyer au spectateur sa propre image. Les lectrices ne vivent donc plus à New York mais au Québec. Afin de rendre ce processus d'identification convaincant à $100 \%$, il fallait ensuite adopter le québécois. Morissonneau remplit ce que Jakobson appelle la fonction conative du langage, " celle qui vise le récepteur. [...] La traduction se doit d'être avant tout "efficace", le mot d'ordre étant de ne jamais "perdre" son public " (Sardin 2004 : 33). Brisset préfère invoquer la fonction phatique, "celle qui garantit que la communication passe bien " (1990 : 228). Concrètement, tous les moyens sont bons pour s'adapter au public et respecter les intentions des auteurs. La variation géographique et la réécriture des tirades avec le régiolecte placent, en fin de compte, l'adaptation dans le prisme de la fidélité. Les deux histoires restent similaires, mais celle des lectrices acquiert une saveur québécoise unique. Le résultat de ce gain est un musical plus drôle pour un public spécifique.

Cette stratégie de traduction est également présente dans les lyrics. Pour des raisons évidentes de place et n'apportant pas de nouveaux éléments d'analyse, nous nous contenterons de le mentionner. Les adaptateurs trouvent ainsi un décalque original qui permet de pimenter le texte cible en allant le chercher non pas dans une simple traduction, mais dans une adaptation personnelle et ludique. D'ailleurs, Morissonneau avoue : " J'étais très fier du résultat "(15).

(14) Rencontre avec Didier Morissonneau et l'auteur de l'article, à Las Vegas, le 12/09/2016.

(15) Idem. 
Ils ne vont toutefois pas s'arrêter là et choisir de jouer la carte de l'humour à fond en insérant de manière éparse des mots québécois dans la version française du roman. Ils ont senti qu'il y avait là une occasion d'ajouter un ressort comique et s'en sont par conséquent donné à cœur joie. Ceci est la deuxième stratégie de traduction :

5 CHRISTIAN. Ew! Anyway, Ana, did you have a chance to look over the contract? (50S-LA p. 34)

5a CHRISTIAN. Ark! Ana, as-tu eu la chance de lire attentivement le contrat ? (50S-Q p. 39)

6 CHRISTIAN. Relax. I would never have had sex with you last night. (50S-LA p. 24)

6a CHRISTIAN. Je n'aurais jamais fourré - fait l'amour - avec vous hier soir. (50S-Q p. 27)

L'humour personnel des auteurs s'observe avec ark et fourré qui sont des termes typiquement québécois ; le premier est une interjection et le deuxième est la traduction de to fuck. II est d'usage de dire que le rire naît d'une surprise. Dans 50 Shades! La Parodie Musicale la surprise vient du décrochage de la part des acteurs. Ils s'échappent pendant quelques secondes de leur rôle en laissant échapper des mots de leur langue, le québécois, alors qu'ils sont supposés parler en français. Ils surprennent ainsi les spectateurs non avertis. Ces petits décrochages n'affectent pas l'histoire, mais lui confèrent un ressort comique original et amusent le public: adapter rime ici avec créativité puisque les traducteurs utilisent leur langue à des fins ludiques. Arrêtons-nous, pour terminer, sur le verbe fourrer. Comme le musical est une parodie d'un livre érotique, il n'est guère surprenant de voir que le livret fourmille d'allusions sexuelles. Les préférences sexuelles de Christian sont protéiformes, mais la domination sadomasochiste reste sa jouissance privilégiée. Afin de le rendre convaincant aux yeux du public, les auteurs emploient, sur le mode humoristique, des termes vulgaires, familiers et tirés du slang [argot]. Rien d'étonnant, donc, de découvrir des ambiguïtés intentionnelles, des calembours et des allusions aux parties sexuelles. Cela n'est pas une mince besogne pour le traducteur : il doit d'abord maîtriser le vocabulaire érotique, puis choisir des équivalents lexicaux qui transmettent l'esprit de l'original et parlent au spectateur. Voici notre troisième stratégie qui est celle de la traduction des termes sexuels par des régionalismes.

"Pour les mots sexuels ou vulgaires, si tu n'es pas dans le régionalisme, ça ne marche pas. Chez nous, au Québec, si tu dis "putain, la meuf je vais la niquer", ça ne veut rien dire. Les gens vont te regarder en se demandant de quoi tu parles. C'est comme en France, si je dis "fourrer la plotte", ça n'a aucun impact. Pour avoir un effet comique et d'écœurement sur le spectateur, il fallait donc que le personnage parle son langage(16). "

Afin de recréer les intentions du texte source et de rendre la fiction érotique aussi vraie que possible, l'adaptateur fait une utilisation par reconnaissance c'est-à-dire qu'il choisit une

(16) Rencontre avec Didier Morissonneau, déjà cité. 
terminologie sexuelle adéquate. Son travail se rapproche ainsi de celui du pornographe puisque les deux mobilisent " des ressources [clarté, réalité, inventivité] qui lui permettent de maintenir l'attention du [spectateur] " (Boulanger 2013 : 56). La chanson "A Red Room of Pain" atteste des audaces jouissives du dominant :

7 CHRISTIAN

CLAMPS UPON YOUR NIPPLES, HOT WAX WITH A BURNING CANDLE [...]

BINDING WITH A SPREADBAR, WITH YOUR HANDS BEHIND YOUR BACK

SUSPENDED FROM THE CEILING HELPLESS WHILE YOUR WORLD GOES BLACK!

VIBRATORS AND DILDOS TOO, I FONDLE YOU WITH PUSSY TOYS

BEAT YOU WITH A RIDING CROP, AND WAKE THE NEIGHBORS WITH YOUR NOISE [...]

AND MY FAVORITE: FISTING! (50S-LA p. 37-38)

7a CHRISTIAN

La canne ou la palette, la ceinture ou bien le fouette ? [...]

Te croquer ou te pincer ou te twister les nipples

Te griffer ou te brûler avec de la cire de chandelle.

Masturbation fellation pénétration flagellation

On a des millions d'options pour maintenir mon érection [...]

Et mon préféré... LE FISTING (50S-Q p. 42)

Ici, I'humour et le sexe font bon ménage. Christian n'est visiblement pas gêné de dire à sa soumise qu'il veut lui procurer du plaisir dans la douleur. Veille doit tenter de rendre ces fantasmes tout en focalisant son attention sur la musicalité de la chanson.

"Quand j'ai traduit cette chanson, j'ai cherché sur Internet un genre de dictionnaire sur tous les outils utilisés dans la pratique sadomasochiste parce que je ne connaissais pas ça vraiment là. La palette est comme une grosse spatule en bois qui sert à taper les fesses. Je l'ai trouvée dans le lexique et je trouvais ça drôle de rimer palette avec fouette qui est la prononciation québécoise de fouet. J'ai écrit -tte pour vraiment accentuer le fait que c'est le mot québécois. Ce que j'ai trouvé drôle avec les nipples, et ça c'était vraiment un effet comique, c'était de faire rimer nipples ['n耳əl] avec chandelle parce que l'acteur dit [Jã.dəl]. Cette rime faisait beaucoup rire. Pour le fisting, je n'ai pas trouvé de mot français, à croire qu'il n'y a que les Anglais qui sont assez fous pour faire ça! Didier m'a donc suggéré de garder le même mot et de l'expliquer sur scène : l'acteur lève son poing dans les airs comme signe de victoire. Quand j'adaptais les chansons, j'allais tout de suite voir Didier. Je les lui chantais et je lui demandais ce qu'il en pensait. II disait : "ça c'est drôle ; ça c'est moins drôle". Et je les retravaillais "(17).

Les explications qu'elle y développe manifestent d'une façon tout à fait juste les théories sur le texte spectaculaire précisant que " le traducteur devrait prendre place aux répétitions du

(17) Skype interview avec Amélie Veille, déjà cité. 
spectacle et être prêt à changer son texte selon les exigences de la scène " (Regattin 2004 : 162). Une collaboration constante est au cœur du travail d'équipe : l'adaptateur accepte de modifier son texte afin de répondre aux attentes du metteur en scène et d'offrir une traduction qui fasse rire le public cible. Dans l'extrait ci-dessus, la surabondance flagrante des pratiques sadomasochistes subit une véritable métamorphose. Chaque mot est minutieusement déniché, traqué et pensé pour répondre, le plus équitablement possible, aux trois critères de Low que sont sense, naturalness et rhyme : certains jeux érotiques sont directement traduisibles en français ; d'autres sont gardés tels quels pour insérer une rime cocasse ; d'autres encore, même s'ils ne possèdent pas d'équivalents, trouvent leur place sur scène. En effet, grâce au "verbo-corps" (Pavis 1990 : 151) qui est l'alliance du mot chanté et du geste, Veille compense les vides lexicaux et révèle les perversions sexuelles du sadique. Elle garde aussi à l'esprit les deux autres critères, singability et rhythm, afin d'installer, le plus naturellement possible, son adaptation sur la musique.

Nous voilà arrivés au terme de cette étude, certes non exhaustive, qui nous permet de conclure que même si l'adaptation d'un musical est une opération complexe en ce qu'elle demande aux traducteurs de la créativité et une solide connaissance en musique, elle se révèle être aussi une " activité jubilatoire " (Desmond 2005 : 81). II faut dire que 50 Shades! The Musical est pour eux un vrai régal car le livret oscille entre humour, références culturelles et sadomasochisme. Afin de retrouver une même intonation, ils décident de s'amuser avec la traduction littérale et de lui donner une saveur québécoise unique. Le québécois est donc un outil de l'efficacité de l'humour puisque les traducteurs s'adressent au public dans l'idiome qui est le sien, améliorent la drôlerie des répliques et tordent les lyrics originaux pour produire des chansons qui sonnent naturelles. Morissonneau et Veille s'approprient ainsi le livret, en maintenant constamment une grande fidélité, pour insuffler leur humour personnel et, de ce fait, contribuer au succès du musical au Québec. II est clair que cette initiative n'est pas guidée par la moindre prétention, mais par la quête d'une création scénique et musicale. En un mot, l'adaptation devient une source d'humour volontaire puisque les traducteurs se laissent aller à leur imagination.

À la lumière de tout cela, une question surgit : le livret peut-il être librement réécrit pour la scène et le public destinataire ? Une réponse à cette interrogation pourrait se trouver dans les théories du texte destiné au spectacle précisant que "l'écriture du spectacle appartiendrait au metteur en scène et aux acteurs " (Regattin 2004 : 162). Le traducteur ne supprime pas le message initial, mais s'en écarte légèrement, au bénéfice d'un jeu créatif avec la langue cible, c'est-àdire plus proche de la première intention des auteurs : faire rire le public. Le livret cible ne fait pas que perdre, il gagne énormément sur les plans comique, musical et chantable.

Alice Defacq est actuellement chercheuse indépendante à Tampa, en Floride. Elle est titulaire d'un doctorat en traductologie de l'Université Catholique de l'Ouest - Collège Doctoral d'Angers (2011). Elle s'intéresse à l'adaptation des musicals anglo-américains pour la scène française. 


\section{Bibliographie}

BOULANGER Pier-Pascale, 2013, "Traduire pour faire jouir ", in Traduire le texte érotique, Montréal (Canada), Figura, p. 43-56.

BOYER Henri, 2001, "L'humour comme connivence intraculturelle et comme obstacle interculturel ", in LAURIAN Anne-Marie, SZENDE Thomas, (dir.), Les mots du rire : comment les traduire? Essais de lexicologie contrastive, Berne, New York et Berlin, Peter Lang, p. 35-41.

BRISSET Anne-Marie, 1990, Sociocritique de la traduction, Théâtre et altérité au Québec (19681988), Longueuil (Québec), Le Préambule.

DESMOND William Olivier, 2005, Paroles de traducteur. De la traduction comme activité jubilatoire, Louvain (Belgique), Peeters.

DI GIOVANNI Elena, 2008, "The American Film Musical in Italy ", in The Translator, 14, p. 295318.

FRANZON Johan, 2008, "Choices in Song Translation ", in The Translator, 14, p. 373-399.

LADMIRAL Jean-René, 2004, "Lever de rideau théorique : quelques esquisses conceptuelles", in Palimpsestes, 16, p. 15-30.

LOW Peter, 2005, "The Pentathlon Approach to Translating Songs ", in GORLÉE Dinda L., (éd.), Song and Significance : Virtues and Vices of Vocal Translation, Amsterdam, Rodopi, p. 185-212.

PAVIS Patrice, 1990, Le théâtre au croisement des cultures, Paris, éd. José Corti.

REGATTIN Fabio, 2004, "Théâtre et traduction : un aperçu du débat théorique ", in L'Annuaire théâtral : revue québécoise d'études théâtrales, 36, p. 156-171.

SARDIN Pascale, 2004, "Les traducteurs sont-ils nécessairement des corsaires ? ", in Palimpsestes, 16, p. 31-43.

SEIDE Stuart, 1982, "La traduction complétée par le jeu ", in Théâtre/Public, 44, p. 60-61.

SOULIER Anne-Marie, 1998, "Traduire entre les lignes ", in Ateliers, 15, p. 11-18.

TRAN-GERVAT Yen-Maï, 2011, "Traduire l'humour ", in Humoresques, 34, p. 5-7.

VENUTI Lawrence, 1995, The Translator's Invisibility, Londres et New York, Routledge.

VENUTI Lawrence, 2006, "Traduction, intertextualité, interprétation ", in Palimpsestes, 18, p. 17-42. 